Portland State University

PDXScholar

4-1-1969

\title{
Steady-State and Transient Currents in Organic Liquids by Injection from a Tunnel Cathode
}

\author{
Pavel Smejtek \\ Portland State University \\ David G. Onn \\ M. Silver
}

Follow this and additional works at: https://pdxscholar.library.pdx.edu/phy_fac

Part of the Physics Commons

Let us know how access to this document benefits you.

\section{Citation Details}

Smejtek, P., Onn, D., and Silver M., Steady-State and Transient Currents in Organic Liquids by Injection from a Tunnel Cathode. Journal of Applied Physics. 40, 2222 (1969).

This Article is brought to you for free and open access. It has been accepted for inclusion in Physics Faculty Publications and Presentations by an authorized administrator of PDXScholar. Please contact us if we can make this document more accessible: pdxscholar@pdx.edu. 


\title{
Steady-State and Transient Currents in Organic Liquids by Injection from a Tunnel Cathode*
}

\author{
M. Silver AND D. G. ONN \\ Physics Department, University of North Carolina at Chapel Hill, Chapel Hill, North Carolina 27514 \\ AND \\ P. SMEJTEK \\ Institute of Macromolecular Chemistry, Czechoslovak Academy of Sciences, Prague, Czechoslovakia
}

(Received 8 April 1968; in final form 13 December 1968)

\begin{abstract}
Experimental data are presented on the currents induced in organic liquids by injection from a tunnel cathode. The injection level was varied over a wide range resulting in almost no space-charge limitation to almost complete space-charge limitation. Results were different from that usually observed in solids, in that at low fields, the steady-state current was proportional to $V^{2}$, while at high fields the current was proportional to $V$. By proper choice of electrode spacing and applied voltage, space-charge-limited current transients as low as $10^{-11} \mathrm{~A} / \mathrm{cm}^{2}$ and 5 sec transit times were observed. A smooth transition between the electrode-limited and the space-charge limited regimes was achieved by varying the junction voltage that varied the injection level.
\end{abstract}

\section{INTRODUCTION}

Transient space-charge-limited currents (TSCLC) were first experimentally and theoretically examined in solids by Many ${ }^{1,2}$ et al. and by Mark and Helfrich. ${ }^{3}$ The former used a pulsed light source to generate a high density of carriers near the electrode, while the latter used a chemically injecting electrode. In both cases, it was assumed that at $t=0$, the density of free carriers at the cathode was so large that, essentially, the field could be considered to be vanishingly small. Further, it was assumed that the TSCLC were too small to deplete the reservoir of carriers.

TSCLC in solids is complicated by the fact that there are trapping levels that slow down the passage of charge through the crystal. This has the effect of reducing the trap-free current by an amount $\theta$, which is the ratio of the free carriers to the trapped carriers. $(\theta$ depends upon the density and distribution of the traps.) If the trapping time is slow compared with a transit time, there will be a slow decay down from the trap-free current to the trap-controlled current. If the trapping time is very fast compared with the transit time, the current rises only to the trap controlled value. The details are clearly presented in the elegant paper by Many. ${ }^{2}$

In liquids, one does not, in general, have the complication of traps for two reasons: (1) The traps are usually impurities that have mobilities comparable to the charge carriers (Argon is a notable exception). ${ }^{4}$ (2) Because of the mobility of the trap, the energy distribution is relatively unimportant. In liquids, one has the possibility of observing either the pure trap-free

\footnotetext{
* Supported in part by the National Science Foundation, Army Research Office Durham, and the Advanced Research Projects Agency.

A. Many, S. Z. Weisz, and M. Simhony, Phys. Rev. 126, 1989 (1962).

A. Many and G. Rakavy, Phys. Rev. 126, 1980 (1962).

3 W. Helfrich and P. Mark, Z. Physik 166, 370 (1962).

4 B. Halpern, J. Lekner, S. A. Rice, and R. Gomer, Phys. Rev. $156,351(1967)$.
}

TSCLC or the very fast trapping case, which behaves in a similar way to the trap-free current. The transport properties then become accessible to measurement and simple interpretation.

The problem with organic liquids has been, until recently, that it was difficult, if not impossible, to make Ohmic contacts to them using standard metal electrodes. This has recently been solved by use of tunnel cathodes. $^{5}$ These electrodes are capable of supplying over $10^{-7} \mathrm{~A} / \mathrm{cm}^{2}$ into vacuum.

These tunnel cathodes have an effect that is different from the usual Ohmic contacts, such as indium on CdS, in that the injection level is limited but variable. Indium on CdS can provide a good Mott and Gurney ${ }^{6}$ cloud for most practical experimental conditions. Because of the high mobility of carriers in CdS, spacecharge-limited currents of $\mathrm{A} / \mathrm{cm}^{2}$ are potentially available. Tunnel junctions, on the other hand, can be used with liquids to give space-charge-limited currents, even though their capabilities are so small because the mobility $\left(10^{-4} \mathrm{~cm}^{2} / \mathrm{V} \cdot \mathrm{sec}\right)$ and consequently the spacecharge-limited currents are also so small.

The variable injection level affords one the opportunity of examining the transition region between electrode and space-charge limitation for its own sake, and also to gain some knowledge about the magnitude of the space-charge cloud at the cathode due to injection.

In this paper we present some experimental results on steady-state and transient currents resulting from injection from these tunnel cathodes. We observe currents both in a high-field region where the current is a linear function of the voltage, and in a low-field region where the current depends upon $V^{2}$. As the injection level is reduced from some high value where the current is space-charge limited, two major effects on the transient are observed; the transit time increases

\footnotetext{
${ }^{5}$ M. Silver, D. G. Onn, P. Smejtek, and K. Masuda, Phys. Rev. Letters 19, 626 (1967).

${ }^{6}$ N. F. Mott and R. W. Gurney, Electronic Processes in Ionic Crystals (Oxford University Press, Oxford, 1948), 2nd ed., p. 169.
} 
Fig. 1. Schematic representation of the apparatus used in these experiments.

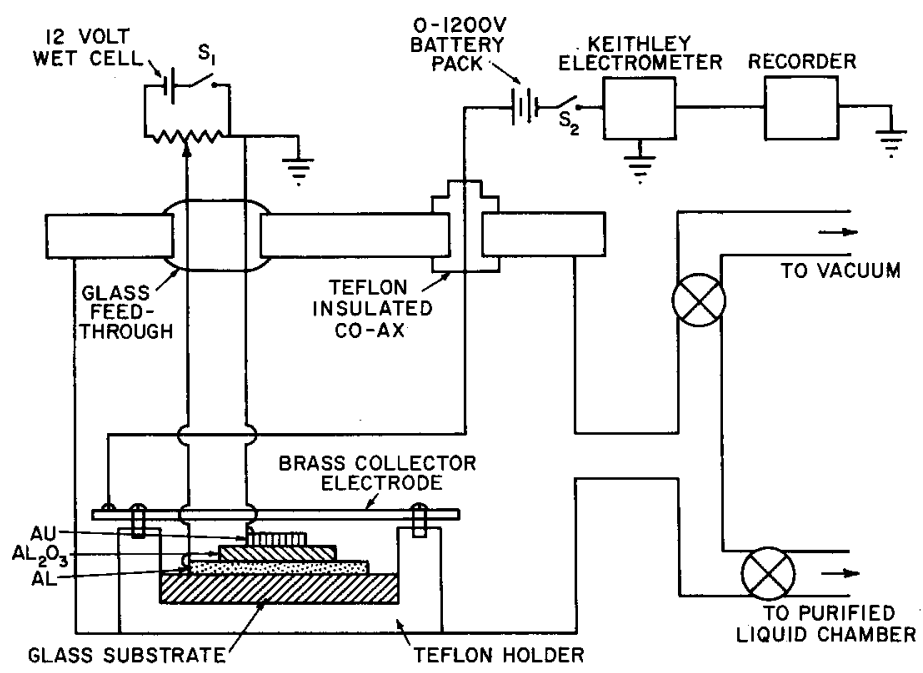

slightly and the current does not rise to as high a peak value. At the high injection levels, it seems that the transient current rises from a very small value at first to a value approximating that predicted by the usual space-charge-limited theory.

\section{EXPERIMENTAL TECHNIQUES AND RESULTS}

The tunnel junctions used were of the $\mathrm{Al}-\mathrm{Al}_{2} \mathrm{O}_{3}-\mathrm{Au}$ type. An excellent survey of their characteristics and operation is given in a review article by Crowell and $\mathrm{Sze}^{7}$ and in another paper by Savoye and Anderson. ${ }^{8}$

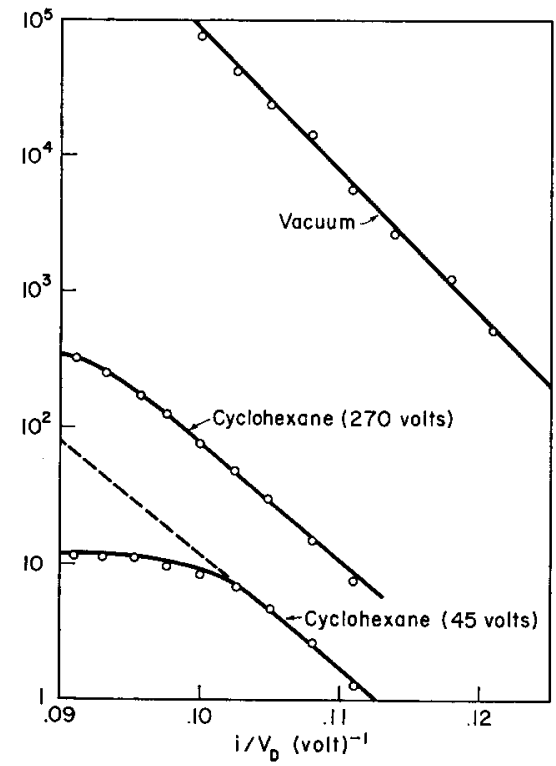

FIG. 2. Log of the emission current vs the reciprocal of the junction voltage for emission into vacuum and cyclohexane for two different collector voltages.

${ }^{7}$ C. R. Crowell and S. M. Sze, Physics of Thin Films (Academic Press, Inc., New York, 1967), Vol. 4, pp. 350-354.

8 E. D. Savoye and D. E. Anderson, J. Appl. Phys. 38, 3245 (1967).
The technique for growing these emitters was exactly the same as that described by Crowell and Sze, 7 and is therefore not repeated here. Our junctions had about 150 A gold layers and about 100-150 $\AA$ oxide layers. These junctions were capable of supplying over $10^{-6}$ $\mathrm{A} / \mathrm{cm}^{2}$ into vacuum for many hours, providing the applied voltage across the oxide during operation was kept below $1 \mathrm{~V} / 13 \AA$ oxide thickness.

A schematic diagram showing the arrangement is shown in Fig. 1. Only standard components were used. A $12 \mathrm{~V}$ wet cell was used to drive the junction, while $45 \mathrm{~V}$ dry cells were used for the collector voltage. A calibrated helipot was used as a voltage divider in the junction voltage circuit. In later experiments, this voltage was measured by a digital voltmeter that was accurate to $\pm 2 \times 10^{-8} \mathrm{~V}$. The switches $S_{1}$ and $S_{2}$ were standard rotary switches, except that the rotor was remounted on Teflon to give better insulation. The dark current was less than $10^{-14} \mathrm{~A}$. With the junction submerged in the liquid, the dark current was less than $10^{-13} \mathrm{~A}$ and was bucked out using the zero adjustment on the Keithley meter.

The collector electrode-junction spacing was typically a few millimeters. The entire structure, diode collector electrode, etc., was mounted in a glass system connected through stopcocks to standard vacuum equipment and to a chamber containing the purified liquids. After the diodes were tested in this chamber in vacuum, the organic liquid was distilled or poured into the chamber until the diode was completely submerged.

Usually ultrapure cyclohexane was used, although some experiments were carried out using benzene. The dark conductivity was usually less than $10^{-16}$ $(\Omega \cdot \mathrm{cm})^{-1}$.

As is well known, ${ }^{7,8}$ the log of the steady-state emission current from these diodes into vacuum plotted against the reciprocal of the junction voltage gives a straight line. The results for one of our diodes are shown in Fig. 2. The injection current can be varied 


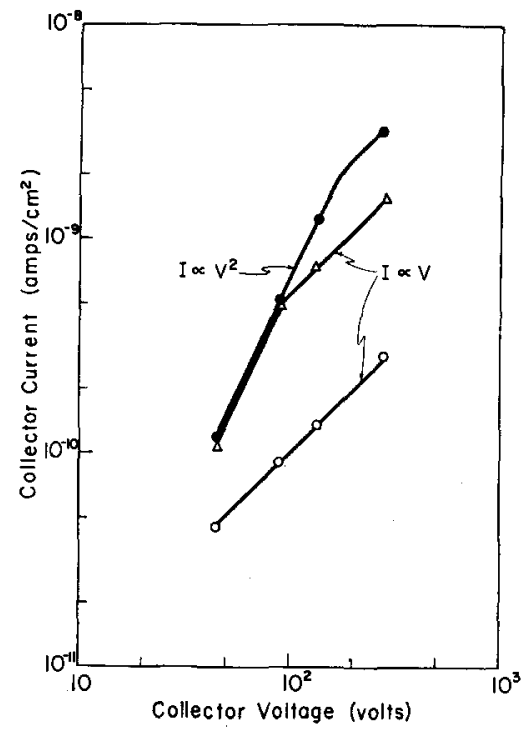

FIG. 3. Log of the emission current vs the $\log$ of the collector voltage for three? different injection levels. The injection level was varied by changing thejjunction voltage and was determined from the data for emission into vacuum at the same junction voltage.

over three decades for only a $2 \mathrm{~V}$ charge in junction voltage.

Also shown in Fig. 2 is the $\log$ of the steady-state current into cyclohexane for the same junction and junction voltage, but different collector voltages. In vacuum, the emission current was independent of collector voltage at voltages above $20 \mathrm{~V}$. However, notice that there is still a voltage dependence at $270 \mathrm{~V}$ for emission into cyclohexane. It should also be noticed that at high injection currents (small $1 / V_{D}$ ), the current into the liquid tends to saturate, while no such tendency is observed for emission into vacuum. Further, it is interesting that although it takes less energy for emission into cyclohexane than into vacuum, the current in the liquid is much less than that available for emission into the vacuum. (The reason that it takes less energy for emission into cyclohexane than into vacuum is that cyclohexane has a positive electron affinity, and therefore the relative work function between gold and cyclohexane is less than between gold and vacuum.)

The log of the current into cyclohexane vs the log of the collector voltage for three different injection levels is shown in Fig. 3. (Injection level is defined as the current available from the junction for emission into the liquid. This is usually controlled by varying the junction voltage. As a crude measure of the injection level, we use the emission current into vacuum at the specified junction voltage.) At low current levels, the current is a linear function of voltage. At the higher injection levels, the current depends upon the square of the voltage at low voltages and then tends toward a linear dependence at high voltages. In the low-voltage high injection level regime, the current is independent of the injection level. This suggests that at low collector voltage but high injection current levels, the current in the liquid is space-charge limited. However, it should also be noticed that there is a large linear region with no indication of a saturation as the collector voltage is increased.

Transient current measurements were made by switching both the junction voltage and the collector voltage. These results are shown in Fig. 4 for three different injection levels but one collector voltage $(180 \mathrm{~V})$. The sharp pulses during the first second or two are just the switching transients. Several interesting features of these transient responses should be noticed: as the injection current is reduced, the transit time (time of the peak current) is increased; the current pulse for the highest injection level resembles those obtained by Many et al.$^{1,2}$; finally, the current at the $2 \mathrm{sec}$ time (the shortest time we can reliably determine the current in these measurements) is less than that predicted by the Many theory. Here the ratio $i_{2 \text { sec }} / i_{\text {peak }}$ is less than $1: 3$, while the theory due to Many predicts a value of around 1:2. This result suggests that the current starts from a very low value as predicted by Lampert and Schilling. ${ }^{9}$

It is not proposed that the Many theory is wrong, but only that one does not have as good an Ohmic contact under these relatively small injection levels as can be obtained from indium on CdS or a pulse of highintensity strongly absorbed light on anthracene or iodine crystals. More is said about this point in the discussion.

As seen from the steady-state measurements, for a given injection, one can come closer to the true spacecharge-limited conditions by lowering the voltage. Transient pulses for the highest injection level available at $135 \mathrm{~V}$ and $0.45 \cdot \mathrm{cm}$ electrode spacing are shown in Fig. 5. The pulse shape appears to be closer to the pure Many-type space-charge-limited current in that the ratio of the peak current to the steady-state current is greater under these conditions than the higher voltage region shown in Fig. 4. The small decaying oscillation after the peak predicted by Many ${ }^{1}$ is beginning to appear. One sees a small minimum at around $6 \frac{1}{2}$ sec and another small maximum at around $8 \mathrm{sec}$. If one takes the transit time to be approximately $4.8 \mathrm{sec}$, these minima and maxima are occurring at $t / t_{0} \simeq 1.1$ and $t / t_{0} \simeq 1.3$, compared with the predicted values of 1.3 and 1.6. This is believed to be reasonable agreement considering the errors in determining the positions of these various maxima and minima.

The transit time, taken to be the peak current value, was measured as a function of the applied voltage for the highest injection level available. The results are shown in Fig. 6. As can be seen, the transit time is a

9 M. Lampert and R. B. Schilling, Phys. Rev. Letters 18, 493 (1967). 
Fig. 4. Transient current response for three different injection levels. The collector voltage in case was $180 \mathrm{~V}$. Upper trace, highest injection level; lowest trace, lowest injection level. The same junction was used for all three traces. The large pulses during the first second or two are due to the switching transient.
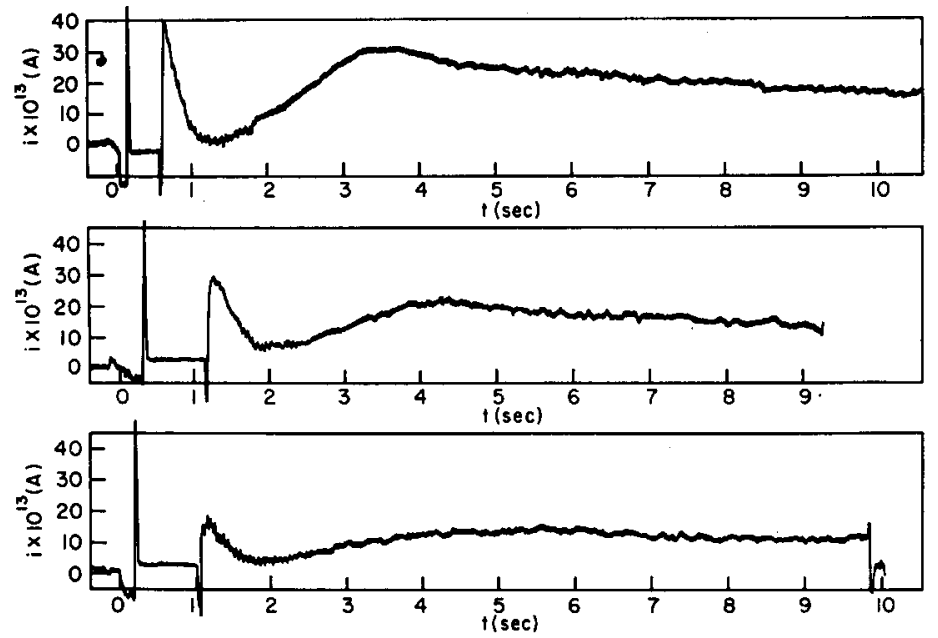

Fig. 5. Transient current response for $135 \mathrm{~V}$ collector voltage. Because of the lower voltage than that used for Fig. 4, the current is closer to a pure space-charge-limited transient.

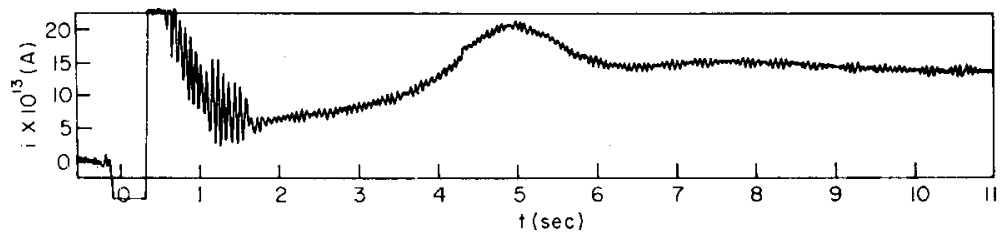

linear function of the reciprocal of the applied voltage. Thus, we are reasonably confident that polarization effect of the liquid-electrode region is not significantly altering the field.

The mobility derived from these transit time measurements is $2.8 \times 10^{-4} \mathrm{~cm}^{2} / \mathrm{V} \cdot \mathrm{sec}$ and is the same value one gets from the steady-state space-charge-limited current $\left(j=\epsilon \mu V^{2} / 8 \pi L^{3}\right)$. This means that the entire geometric area of the junction is emitting at least at high injection currents where space-charge limitations are evident.

\section{DISCUSSION}

The main features of the experimental results presented here can be explained if it is assumed that the density of charge near the cathode region is determined primarily by the magnitude of the injection current and back diffusion into the injecting electrode. Such an

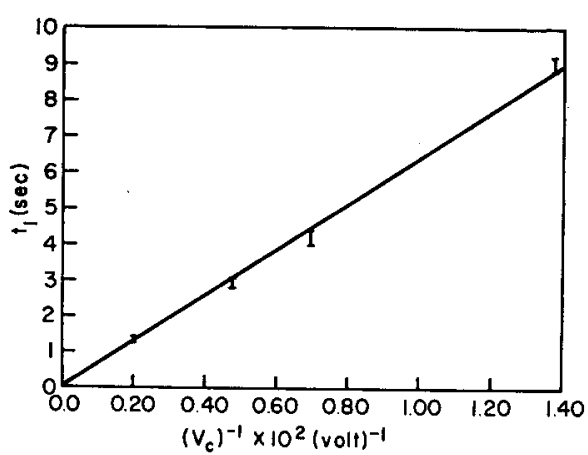

FIG. 6. Transient time vs reciprocal applied voltage. assumption is not unreasonable and was considered by Thomson ${ }^{10}$ for the problem of photoinjection into a dense gas, and by Mott and Gurney ${ }^{6}$ for injection into an insulator from a thermionic metal contact. Lampert ${ }^{9}$ used the same idea to show that the initial value of the transient space-charge-limited current is small rather than very large as expected from diffusion of the physically unrealistic infinite density reservoir.

As first shown by Thomson, ${ }^{10}$ the steady-state current for free electrons and no space-charge distortion is

$$
j=j_{e}\left[1+v_{t h} /(6 \pi)^{1 / 2} \mu E\right]^{-1} \simeq j_{e}(6 \pi)^{1 / 2} \mu E / v_{t h},
$$

since $v_{t h} \gg \mu E$, and where $j_{e}$ is the emission current, $v_{t h}$ the thermal velocity, $\mu$ the mobility, and $E$ the applied electric field. Mott and Gurney calculated their spacecharge cloud from continuity of current for free electrons and arrived at the well-known formula

$$
\rho(x)=\rho_{0}\left(1+x / x_{0}\right)^{-2},
$$

where

$$
\rho_{0}=j_{e}(6 \pi)^{1 / 2} / v_{t h} \text { and } x_{0}=\left(2 \pi \rho_{0} e / \epsilon k T\right)^{-1 / 2} .
$$

In most liquids, it is unlikely that free electrons exist for more than $10^{-9} \mathrm{sec}$, but rather they form lowmobility negative ions or solvated electrons. If this relaxation is fast so that the diffusion length of the free electron during its lifetime is small, Eqs. (1) and (2) are only slightly altered. It is simple to show, using $-\nabla \cdot j_{f}-\rho_{f} / \tau=0$ and $-\nabla \cdot J_{s}+\rho_{f} / \tau=0$, where the subscripts $f$ and $s$ refer to the free electron and the slow

${ }^{10} \mathrm{~J}$. J. Thomson and G. P. Thomson, Conduction of Electricity Through Gases (Cambridge University Press, New York, 1928),
3rd ed., Vol. 1, p. 466. 


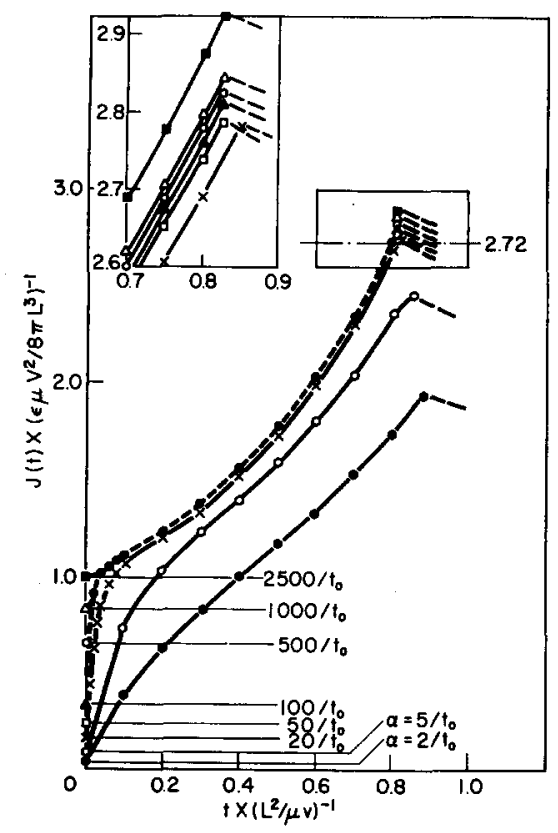

Fig. 7. Theoretical shape of the transient current pulse for various injection levels $\alpha$ in units of the reciprocal of the spacecharge free transit time. The region near the peak current is expanded and shown in the upper left-hand corner. Also shown are the initial values of the current at $t=0$ for different injection levels.

ion or solvated state, respectively, that

$$
j \simeq j_{e}(\pi / 6)^{1 / 2} e E \lambda / k T,
$$

where $\lambda$ is the mean free path for elastic scattering, and

$$
\left.\rho(x)\right|_{x>\delta} \simeq \rho_{0}\left[1+(x-\delta) /\left(x_{0}-\delta\right)\right]^{-2},
$$

where $\delta$ is the diffusion length of the free electrons during their lifetime.

Either Eq. (1) or (3) shows the linear dependence of the current upon field strength in agreement with experiment.

The transient current during the first transit time can be calculated simply by including a nonzero field at the cathode. In this case, the Many ${ }^{2}$ equation for the current is modified as follows:

$$
J(t)=(\epsilon \mu / 8 \pi L)\left[E_{L}^{2}-E_{0}^{2}\right]
$$

This is the same equation used by Weisz et al. ${ }^{11}$ to calculate the effect of a time-dependent reservoir.

The difference between $E_{L}$ and $E_{0}$ is just the total charge in the volume, i.e.,

$$
E_{L}-E_{0}=\int_{0}^{t} \alpha E_{0} d t+(4 \pi / \epsilon) \rho_{0} x_{0}
$$

where $\alpha$ is $4 \pi / \epsilon$ times the current per unit field strength that can be extracted from the reservoir $[(4 \pi / \epsilon) j / E$ from Eqs. (1) and (3)].

Equations (5) and (6) have been solved numerically for different values of $\alpha$. These results are shown in Fig. 7 where the values of $\alpha$ are expressed in terms of the reciprocal of the transit time. One sees a smooth transition from the small space-charge distortion case to the almost complete space-charge-limited current. These curves show the same features as the experimental results shown in Fig. 4 for various injection levels. The initial value of the current at $t=0$ increases smoothly with increased injection level and saturates at the Many value.

A slight overshoot from the maximum value of the current and a slightly longer transit time than that predicted by Many $\left(j / j_{0}=2.72\right)$ is evident, and is due to the contribution to the current by that part of the Mott and Gurney cloud where the density is less than $C V / L$. [The maximum current during the transient is attained (as shown by Many ${ }^{2}$ ) when that charge density approximately equal to $C V / L$ reaches the anode.] The overshoot region is expanded to show it in more detail. The experimental results in Fig. 5 show this overshoot as well. The ratio of peak current to the steady-state current predicted by Many $^{2}$ is 1.21 , while one obtains a value of around 1.35 from Fig. 5 .

If in experiments such as these $\alpha$ can be determined, then one can obtain information on the mean free path for elastic scattering for electrons in liquids that have not been determined before. More careful measurements near $t=0$ are needed than have been made here.

\section{ACKNOWLEDGMENTS}

The authors are grateful to Dr. George Basbas for his invaluable help with the numerical analysis and to Dr. S. I. Choi for many useful discussions.

\footnotetext{
${ }^{11}$ S. Z. Weisz, A. Cobas, S. Trester, and A. Many, J. Appl. Phys. 39, 2296 (1968).
} 